\title{
Professionnalité des enseignants: VERS UNE DÉFINITION DU COLLECTIF À PARTIR D'UNE ReCherche au Lycée Autogéré de Paris (LAP)
}

Maria Papantoniou ${ }^{\star}$

\begin{abstract}
RÉSUMÉ
Nous proposons de présenter dans cet article une partie des résultats de la recherche que nous avons effectuée au sein du Lycée Autogéré de Paris (LAP). Nos observations sont centrées sur le fonctionnement de ce lycée et ses incidences sur le rapport des élèves à l'école et aux disciplines scolaires. Nous avons été amenée à proposer le concept de Travail d'Institutionnalisation (TDI) pour désigner la pratique d'autogestion au LAP qui consiste en un double travail: d'une part, sur les normes internes du lycée et, d'autre part, sur ses rapports aux institutions qui le traversent. Comment ce concept interroge-t-il la "violence" qui surgirait dans l'institution éducative et les pratiques des professionnels censées y répondre?

Mots-clés: institution; Lycée Autogéré de Paris; normativité; collectif.

\section{Professionalism of teachers:}

\section{TOWARDS A DEFINITION OF THE COLLECTIVE BASED ON A Research on the Autogestioned LyCEum of PARIS (LAP)}

\begin{abstract}
We propose to present in this article some results of the research that we conducted in the Autogestioned Lyceum of Paris (LAP). Our observations focus on the functioning of the school and its impact on the relationship between students, school and school subjects. We were led to propose the concept of Institutionalization Work $(I W)$ to refer to the practice of autogestion at the LAP which is a double task: on the one hand concerning the internal norms of the school and on the other hand concerning its relationship to institutions that flow through it. How this concept helps us to examine and understand "violence" that may arise in the educational institution and subsequent professional practices?
\end{abstract}

Keywords: institution; Autogestioned Lyceum of Paris; normativity; collective.

\footnotetext{
^ Docteur en Sciences de l'éducation (Paris 8, 2010), membre du Laboratoire Cultures, Éducation, Sociétés (LACES), EA 4140, Université de Bordeaux. Adresse: 12 rue des Gants, 33000 Bordeaux. E-mail: maria.papantoniou.maria@gmail.com
} 


\section{Notre POINT DE DÉPART: LA PÉDAGOGIE ET L’ANALYSE INSTITUTIONNELLES}

Boumard et Marchat (1994) se sont intéressés au chahut. Cette pratique "déviante" des élèves est, selon les auteurs, liée à la forme "classe", qui est elle-même historiquement liée à l'institutionnalisation de l'école républicaine. D'après eux, le chahut, qui est pour les élèves une manière de questionner de manière ludique l'école (et tout d'abord l'interdiction du jeu en son sein) pose des difficultés aux enseignants qui le perçoivent comme une critique leur étant personnellement adressée. Tout se passe comme si, une fois de l'autre côté de la barrière, les élèves devenus enseignants perdaient leur sens de l'humour tout autant que leur faculté critique. Seule une pédagogie qui permet et qui valorise l'analyse de l'institution peut restituer aux enseignants leurs capacités critiques: c'est à eux d'inviter les élèves à une analyse de ce qui se trouve interrogé dans et par le chahut. Le chahut n'est un "analyseur" que si les enseignants savent s'en saisir. La formation des enseignants, le pédagogisme, les didactiques sont en cause. Ils occultent cette dimension (la dimension institutionnelle) de l'enseignement.

Ces considérations résument bien à notre avis les points de vue de la pédagogie institutionnelle, de l'Analyse Institutionnelle (AI) ainsi que de la pédagogie lapienne. Mais une question reste en suspens. Comment se forme-t-on à l'analyse institutionnelle? Quels types d'apprentissages contribuent à sensibiliser les enseignants et les autres praticiens aux dimensions institutionnelles des problèmes qui se posent dans leurs pratiques? L'AI possède-t-elle un "modèle de professionnalité" dont elle se sert? Quel est le noyau central des diverses pratiques se définissant comme institutionnelles ou institutionnalistes? Et, surtout, d'où viendrait-il?

Selon Ardoino et Lourau (1994, p. 6-7), les approches du groupe et de la dynamique des groupes se trouvent aux origines théoriques de la pédagogie et de l'analyse institutionnelles. Ces mêmes auteurs soulignent aussi les influences de la pédagogie libertaire et de la tradition anarchiste. De notre côté, nous avons tenté de consolider dans notre thèse notre concept de Travail d'Institutionnalisation (TDI) en recourant à une analyse des pratiques du collectif issues de la tradition anarchiste. Les diverses dimensions et les effets de la mise en place d'un tel travail que nous avions observés au LAP devraient être examinés dans d'autres contextes, dans d'autres collectifs. Pour ce faire, nous avons exploité des éléments d'analyse dans l'observation de deux collectifs autogérés du milieu anarchiste de Thessalonique, une station radio et une revue.

Cette observation nous a permis de confirmer la principale caractéristique de ce travail, c'est-à-dire le fait qu'il s'agit d'un double travail: travail sur ce qui se trame à l'intérieur du collectif et travail sur les différents liens entre le collectif et "l'extérieur". Cela nous a entraînée sur une piste: le collectif, le fonctionnement collectif (élément central de toute pratique d'autogestion, y compris celle du LAP) invite et oblige à analyser l'institution. Comment décider, comment faire collectivement, si les questions sur nos implications institutionnelles ne sont pas posées? Avant d'être une discipline universitaire ou un cadre théorique, l'Analyse Institutionnelle est une pratique sociale. 


\section{LE NORMATIF, LE MILIEU}

Une autre caractéristique de ce que nous avons nommé Travail d'Institutionnalisation est qu'il fait son apparition à chaque fois que les "manières habituelles de faire" sont soit mises en cause (par une contestation, un chahut, une déviance, une perturbation...) soit insuffisantes pour traiter une nouvelle problématique qui apparaît et qui se pose au collectif. La question de la norme s'est donc posée dans notre travail et le recours à Canguilhem a répondu à notre curiosité. Pour Canguilhem (1966, p. 77), est normal ce qui est normatif et est normatif ce qui institue des normes. Qu'y a-t-il de plus apparent? Le collectif qui se livre au TDI est en train d'instituer des normes, d'effectuer un travail normatif.

Pour Canguilhem, un organisme vivant est normatif dans son milieu. Selon lui, la normalité et le normatif ne sont pas à envisager en dehors de ce qui entoure l'organisme, c'est-à-dire son milieu. Le travail normatif que nous avons voulu attribuer au collectif étant un TDI qui se fait toujours et de manière complémentariste sur l'intérieur et l'extérieur, notre rapprochement peut tenir, mais seulement à la condition de définir qui est normatif et relativement à quel milieu.

Pour nous, celui qui est normatif ici est le collectif relativement à un milieu de relations qu'il entretient avec "l'extérieur" et qui "delimite" son milieu. Nous avons décrit, à propos des trois collectifs sur lesquels nous avons travaillé, comment ce milieu se dessine à partir des relations qu'il noue avec des institutions ou des collectifs (certains plus en lien avec "la tache", d'autres plus orientés par des connivences). Mais qu'en est- il de l'individu et de ses propres rapports aux normes?

La question est d'autant plus importante que certains travaux en sciences de l'éducation accordent de leur côté une importance à la normativité à laquelle les élèves en difficulté peinent à accéder. Dans leur travail sur les dispositifs relais, Martin et Bonnéry (2002) distinguent trois catégories de parcours d'élèves dans les dispositifs selon qu'ils arrivent ou non à rétablir un rapport plus adéquat aux normes (pour leur réussite scolaire). Même si la description des catégoriestypes est convaincante, nous regrettons qu'aucune mention ne soit faite aux processus de construction de ce rapport aux normes et qu'aucune hypothèse ne soit formulée sur les effets éventuels des dispositifs étudiés sur ce processus. Les auteurs auraient-ils oublié le complément nécessaire du normatif, c'est-à-dire le milieu ? Le glissement dans les termes, le passage du normatif à la normativité, implique une mise en abîme du fait que le normatif est ce qui institue des normes. La normativité serait un bon rapport aux normes. Mais quelles normes? Où est passé le normatif, le milieu?

De notre côté, dans la topique que nous avons voulu esquisser en parlant de TDI au niveau du collectif, nous avons donné à l'individu une place de membre du collectif qui participe au collectif et qui participe à ce travail en même temps qu'il l'observe. Les normes du collectif s'élaborent dans le 
collectif qui n'est qu'une partie du milieu de l'individu. En même temps, les normes sont "métabolisées" et les rapports des individus aux normes sont élaborés (et pas seulement celles du collectif).

\section{L'INDIVIDU, LE GROUPE ET LEURS ANALYSES}

En nous basant sur la monographie d'un élève du LAP, nous avons essayé de décrire les effets "du collectif" sur l'individu. Rappelons que, pour nous, le collectif mène un TDI et que l'individu, membre du collectif, participe à ce travail tout en l'observant.

Il nous semble qu'une phrase de Jean-Claude Rouchy (1998, p. 165) décrit bien ce que le collectif "offre" à l'individu:

L'individuation n'est jamais achevée. C'est un processus groupal qui dépend de trois facteurs déterminants pour qu'il ait un effet positif: que le groupe soit contenant, présente un espace transitionnel (où les échanges favorisent par la parole la mentalisation des sensations et des sentiments), et propose des images identificatoires enrichissantes pour la structuration du Moi.

Et ailleurs:

L'espace analytique du groupe présente une dimension thérapeutique très spécifique: il délimite un intérieur et un extérieur mettant en rapport l'intrapsychique et l'histoire du sujet avec l'intersubjectif et le rapport à la réalité du groupe de travail. Le dispositif à la fois psychanalytique et psychosocial a trois dimensions principales: l'aspect contenant d'angoisses primaires, l'espace transitionnel "aire intermédiaire d'expression", lieu de métabolisation de la réalité psychique et de la réalité externe, et des processus identificatoires offrant de nouvelles possibilités de structuration du sujet (ROUCHY, 1998, p. 283).

Pourquoi ces propos de cet analyste des groupes ont-ils attiré notre attention? Nous avions d'abord le sentiment qu'ils décrivaient bien ce que nous avions vécu dans des groupes ainsi que ce que nous avions décrit en d'autres termes et avec d'autres références. Si les références de Rouchy sont le groupe de travail, le concept de contenant-contenu de Bion et l'aire transitionnelle de Winnicott, nous avons fait référence à Devereux pour parler du "dedans" et du "dehors" et à Gilbert Simondon pour parler de ce qui lie l'individu au groupe et au social.

Pour Simondon (1989, p. 18),

il serait possible de considérer tout véritable relation comme ayant rang d'être, et comme se développant à l'intérieur d'une individuation nouvelle; la relation ne jaillit pas entre deux termes qui seraient déjà des individus; elle est un aspect 
de la résonance interne d'un système d'individuation; elle fait partie d'un état de système.

Si la relation a rang d'être, c'est la relation entre individu et groupe qui donne rang d'être au groupe. Les individuations, de l'individu et du groupe, sont un système d'individuation en résonance interne. Le groupe serait un intermédiaire dans la relation de l'individu au social, un espace dans lequel se métabolisent la réalité psychique et la réalité (externe) du social. Le groupe étant contenant pour le sujet, le sujet pourra contenir ses angoisses. Le groupe étant un espace transitionnel permettra à l'individu d'accéder à autre chose que le groupe.

Dans notre monographie de Roman (élève du LAP), nous avons décrit comment l'observation du collectif et du TDI lui avaient permis un double mouvement: de renonciation à ses pratiques d'évitement du travail scolaire et d'acceptation d'une réconciliation avec une partie de ce qui venant de l'extérieur lui paraissait d'abord comme une norme aberrante puis comme une contrainte structurante. Dans notre analyse, le collectif, espace intermédiaire, contenant et transitionnel, offre aussi à ses membres un "spectacle" d" "individuation en acte" que l'individu observe et dont il s'inspire pour sa propre individuation.

\section{LES ESPACES INTERMÉDIAIRES: UNE APPROCHE SOCIOLOGIQUE. \\ LES COLLECTIFS ET LES INSTITUTIONS}

Le fil conducteur de notre travail est une tentative de saisir le collectif en tant qu'instance qui permet aux individus un accès à l'analyse de l'institution, un accès à la compréhension de la dimension institutionnelle de leurs activités collectives et individuelles. C'est en suivant ce fil que nous proposons une lecture du concept d'espaces intermédiaires. Nous nous intéresserons donc au travail de Laurence Roulleau-Berger sur les espaces intermédiaires dans l'optique d'y rechercher si dans ce qu'elle nomme espaces intermédiaires nous pourrions déceler des similitudes avec les caractéristiques que nous considérons comme des caractéristiques du "collectif".

Décrire des collectifs n'est pas le but de l'auteur, qui enquête sur les stratégies des jeunes en insertion professionnelle face aux dispositifs publics d'insertion. C'est dans le choix de son terrain de recherche que nous pouvons déceler dans un premier temps et de manière très simple un lien avec le collectif. La population des jeunes sur lesquels elle enquête est double: d'une part, des jeunes en lien d'adhésion avec des dispositifs et, d'autre part, des "micro-lieux", lieux "de mise en cuvre de stratégies d'évitement [où les jeunes] pratiquent des activités artistiques [...] et qui apparaissent souvent comme des prétextes pour agir collectivement" (ROULLEAU-BERGER, 1993, p. 193-194).

Les espaces de recomposition sociale que traite la sociologue dans un premier temps se forment dans les dispositifs où l'adhésion des jeunes est forte, qui deviennent des lieux "d'échanges et d'apprentissages collectifs" et où le travail s'effectue sur la base de projets. Dans les Missions Locales, qui favorisent ces aspects, des propositions des stages (liées à des projets par exemple) peuvent 
aboutir, alors que ce ne serait pas le cas pour les mêmes propositions émises par le Pôle Emploi. On ne s'étonnera pas de voir là "où le mode d'insertion ne correspond pas toujours à celui qui est attendu du point de vue des acteurs liés aux politiques sociales et aux marchés du travail" (ROULLEAU-BERGER, 1993, p. 205) l'apparition des notions d'adhésion et de projet qui permettent d'esquisser un "minimum" d'espace collectif. Certes, les jeunes et les acteurs de ces dispositifs sont loin de former ensemble un collectif. Mais la demande d'adhésion de la part de ces dispositifs des Missions Locales, les idées de lier insertion sociale et professionnelle et de travailler autour des projets, les discussions collectives que ces idées entraînent entre les jeunes et la diversité des acteurs introduisent malgré tout dans ces espaces (ne serait-ce que faiblement) des éléments forts du collectif: la libre adhésion et le projet collectif. Les idées "humanistes" qui présideraient les projets Schwartz seraient-elles plutôt "collectivistes"?

Les espaces de création culturelle sont quant à eux des "lieux" créés et gérés par des jeunes. Les buts précis de la recherche en question ne nous permettent pas de retrouver dans cet article beaucoup d'éléments sur la gestion et le fonctionnement de ces "lieux". Mais on comprend qu'ils sont créés et gérés par les jeunes et qu'ils apparaissent "comme prétexte d'agir collectivement" (ROULLEAU-BERGER, 1993, p. 194). L'auteur parlera d'espaces "moins visibles... très peu liés aux institutions" (ROULLEAU-BERGER, 1993, p. 191). Dans ces espaces, on constate des stratégies d'évitement, de ruse: les jeunes

évitent, adhèrent faiblement aux dispositifs d'insertion [...]; ils peuvent ruser avec ces dispositifs, par exemple, en créant une association 1901 avec des statuts fictifs, des contrats emploi-solidarité par exemple, pour en faire bénéficier "des copains" (ROULLEAU-BERGER, 1993, p. 194-195).

Nous pouvons nous demander si l'évitement, la faible adhésion, la ruse ne témoignent pas d'un lien plutôt que de l'absence de lien aux institutions auxquelles ils s'adressent. Gérer les contrats emploi-solidarité ne serait-il pas le but assigné à ces institutions d'insertion? Et que dire de l'institution du travail lorsque dans ces espaces "les stratégies de ruse, d'évitement des politiques sociales tendent à donner lieu à des parcours de réinvention du travail" (ROULLEAUBERGER, 1993, p. 198, c'est nous qui soulignons)?

À côté de ce choix de définir ces espaces comme de "formes non institutionnelles" ou comme des formes "qui occupent des interstices sociétaux à côté des formes plus institutionnelles qui constituent la société" (ROULLEAU-BERGER, 2003, p. 151) les qualités "instituantes" attribuées à ces espaces peuvent nous paraître quelque peu exagérées. Ces espaces se présentent comme "des lieux de redéfinition d'ordres et de desordres locaux" (ROULLEAU-BERGER, 1994, p. 880). Ces espaces sont les produits de "résistances collectives au processus de précarisation salariale". Ils "travaillent l'espace public de manière discrète et continue" et traduisent "des formes de civisme revendicatif" (ROULLEAU-BERGER, 2003, p. 151). Dans ces espaces, les individus: 
tendent à diminuer le risque d'échec... réapprendre l'estime de soi $[\ldots]$, valoriser mutuellement les expériences des uns et des autres [...] Les individus revendiquent le droit de ne pas accepter un emploi dans lequel le travail apparaît disqualifiant, le droit d'improviser [...] (ROULLEAUBRGER, 2003, p. 152).

Plus encore: dans ces espaces:

se fabriquent des compétences et de 'l'intelligence collective'... les compétences collectives activent, voire transforment, les savoirs et compétences individuelles pour les légitimer dans des espaces de travail non directement liés au salariat (ROULLEAU-BERGER, 2003, p. 152).

Ces espaces ne joueraient donc pas seulement un rôle dans et à côté de ce qui s'appelle politiques publiques d'insertion et dans et à côté du travail et de sa valorisation, mais aussi dans et à côté d'une autre institution, celle du salariat. On n'aurait pas tort de dire qu'un grand poids semble peser sur les épaules de ces espaces!

Nous avons le même sentiment qu'une lourde tâche pèse sur les épaules de quelques-uns quand le niveau international est abordé. Dans un contexte où "la société mondiale du risque rend impraticables les normes de prévision et de contrôle des conséquences futures de l'action humaine" (ROULLEAU-BERGER, 2003, p. 153), les exemples du commerce équitable ou des organismes de finance solidaire sont évoqués parce qu'ils "montrent très bien comment des réseaux de solidarité internationale se développent autour de la gestion de ces risques d'exclusion à l'échelle mondiale" (ROULLEAU-BERGER, 2003, p. 153).

\section{LE COLLECTIF, LE PROJET}

En ce qui concerne la question du collectif qui nous préoccupe ici, les définitions de Roulleau-Berger qui transparaissent dans ces textes sont quelque peu éloignées des nôtres. La sociologue parle en termes de "jeunes" ou de "groupe" quand elle cite par exemple une association 1901 fondée par des jeunes. Le terme de collectif est utilisé quand il est question des agencements entre "groupe" des jeunes et représentants des politiques publiques d'insertion et des négociations entre eux autour d'un "projet" (ROULLEAU-BERGER, 1993). Nous partageons avec l'auteur la centralité qu'elle accorde aux négociations et à la notion de projet. Nous conservons au contraire quelques doutes aussi bien sur la localisation du collectif que sur la définition du projet.

Un collectif est effectivement un lieu dans lequel un projet se négocie en permanence. Le projet est, quant à lui, de notre point de vue, la question que le collectif se fixe comme sa question centrale et qui va par la suite constituer une tâche autour de laquelle vont s'organiser les différentes autres tâches particulières. Les négociations au sein du collectif porteront autour de cette "tache" centrale et autour des différentes divisions que les tentatives d'accomplissement de cette tâche impliqueront pour le collectif, à l'intérieur de celui-ci mais aussi 
avec l'extérieur. Nous ne parlons donc pas en termes d'adéquation entre fins et moyens. Bien évidemment, dans un collectif, le choix des moyens mobilisés peut donner lieu à des réalisations différentes mais ce qui importe, c'est le lien entre ces réalisations et le projet qui se négocie. Quand les réalisations s'éloignent sensiblement du projet initial, ce projet peut être abandonné. Dans ce cas, le collectif peut s'auto-dissoudre ou se donner un autre projet.

C'est la notion de projet qui peut nous guider pour localiser le collectif. Quand des acteurs de l'insertion et un "groupe" des jeunes commencent à négocier autour d'un projet, de quel projet parle-t-on? De celui des jeunes, de celui des acteurs de l'insertion ou bien de celui sur lequel ils vont négocier? S'il s'agit de ce dernier, quel rapport aura ce projet avec les projets (il faudrait plutôt dire les aspirations, étant donné qu'on ne sait pas si ces projets existent vraiment) qui se trouvent au centre des préoccupations des "groupes" des jeunes et au centre des préoccupations des équipes des professionnels de l'insertion? Nous pensons qu'il vaut mieux parler de négociations entre un collectif des jeunes et quelques professionnels qui formeraient une équipe de travail. Pour nous, le collectif se situerait plutôt au niveau du "groupe" de jeunes. Un collectif plus large incluant le groupe des jeunes et des professionnels ne nécessiterait pas seulement de travailler sur une réalisation concrète mais aussi de se donner ensemble un projet central à leur existence en tant que collectif, un projet ayant les caractéristiques que nous avons précédemment décrites.

\section{Pour conclure. Que Cherche-T-on?}

Lors de nos recherches sur le LAP et les collectifs autogérés, nous avons cherché une définition du collectif. Le collectif a un projet autour duquel il mène un TDI sur ce qu'il institue et sur les institutions qui le traversent. Cette définition est née d'observations sur des collectifs autogérés, l'accent étant mis sur quelques principes de fonctionnement qui s'associent au choix de l'autogestion, les principes de libre association, de gestion collective, d'analyse permanente du fonctionnement, de la tâche et des divisions du travail que nous avons analysés dans leurs interactions. Mais en dehors des collectifs se définissant comme autogérés qu'en est-il du "collectif'? S'il existe des jeunes ou moins jeunes qui cherchent un "prétexte d'agir collectivement", que cherchent-ils effectivement?

Nous avons pensé qu'il serait plus pertinent de chercher "le collectif" dans ce qui se produit quand des individus aspirent à travailler collectivement plutôt que de limiter nos investigations à l'application des principes de fonctionnement. Nous constatons que l'aspiration d'agir collectivement implique souvent l'aspiration d'une équité face à la gestion. À la gestion de quoi? Du projet, de la tâche, que ces individus se sont donné ensemble. L'aspiration d'équité entraîne avec elles les idées de libre association et de gestion collective. Mais, à notre avis, dès que la gestion collective se met en place, c'est la transversalité qui fait immanquablement son apparition. Le collectif et les individus (membres du collectif) sont traversés 
(par le biais des appartenances) par des institutions, ils sont impliqués. Les analyses nécessaires autour de la tâche convoqueront l'analyse de ces implications, de la transversalité, des institutions qui sont là, qu'on le veuille ou non.

Boumard et Marchat, que nous avons cités au début de cet article, insistent sur la difficulté des enseignants à analyser précisément les dimensions institutionnelles qui logent dans nos pratiques. La pratique du "collectif" serait, à notre avis, une bonne école pour ceux qui aspirent à analyser les dimensions institutionnelles de leurs pratiques professionnelles. Nous pensons effectivement que c'est par le biais de cette pratique que se forment les praticiens de l'Analyse Institutionnelle. En tout cas, la conclusion de notre recherche sur la pédagogie lapienne pourrait se résumer par l'idée que sa spécificité se trouve dans sa pratique du collectif.

Pour clore ce texte, nous aimerions citer une "recommandation" venant d'un chercheur en Sciences de l'éducation, Jean-Yves Rochex. Dans la série des documents vidéo "Connaissance et Savoir", "Les grands témoins de l'éducation", Rochex répond aux questions de Philippe Merieu avant de se soumettre à l'épreuve des questions des étudiants en IUFM. Un grand nombre de questions sont posées, qui obtiennent des réponses diverses. Mais les étudiants semblent obstinés, ils insistent comme s'ils voulaient demander: Que faire en fin de compte? Comment un chercheur se sortira-t-il de l'embarras d'une telle question? Estil capable ou "legitime" d'y répondre? Et au chercheur, qui a bien voulu jouer le jeu de questions-réponses, de répondre: "Chercher le collectif"...

À un moment où des injonctions d'autonomie et de responsabilité ainsi que des injonctions de partenariat et de "travail en equipe" envahissent les professionnels de l'éducation, il nous paraît important de chercher à définir ce qu'est le collectif et de se demander ce qui empêche le travail en équipe d'être un vrai travail collectif. Nous souhaitons avoir l'occasion de creuser cette question dans des recherches futures.

\section{RÉFÉRENCES}

ARDOINO, J.; LOURAU, R. Les pédagogies institutionnelles. Paris: PUF, 1994.

BOUMARD, P.; MARCHAT, J. F. Chahuts: ordre et désordre dans l'institution éducative. Paris: A. Colin, 1994.

CANGUILHEM, G. Le normal et le pathologique. Paris: PUF, 1996.

MARTIN, E.; BONNERY, S. Les classes-relais: un dispositif pour les élèves en rupture avec l'école. Paris: ESF, 2002.

ROCHEX, J. Y. Le rapport au savoir: comprendre l'échec scolaire, entretien avec Philippe Merieu. Connaissance et Savoir, Les grands témoins de l'éducation, 2010 . 
ROUCHY, J. C. Le Groupe, espace analytique. Paris: Erès, 1998.

ROULLEAU-BERGER, L. La construction sociale des espaces intermédiaires: l'exemple de jeunes en emploi précaire face aux politiques sociales. Sociétés contemporaines, n. 14/15, p. 191-209, 1993.

ROULLEAU-BERGER, L. Ordres et désordres locaux: des politiques d'insertion aux espaces intermédiaires. Revue française de science politique, $44^{\mathrm{e}}$ année, n. 5 , p. 856-880, 1994.

ROULLEAU-BERGER, L. La production d'espaces intermédiaires. Hermès, n. 36, p. 147-156, 2003.

SIMONDON, G. L'individuation psychique et collective. Paris: Aubier, 1989.

Reçu: Octobre 15, 2012 Accepté: Novembre 17, 2012 